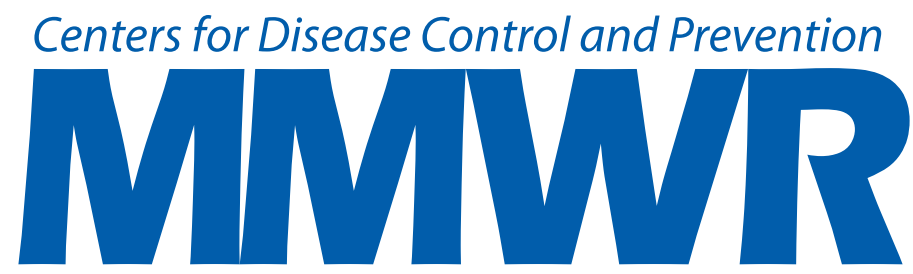

Weekly / Vol. 67 / No. 45
Morbidity and Mortality Weekly Report

November 16, 2018

\title{
Suicide Rates by Major Occupational Group - 17 States, 2012 and 2015
}

\author{
Cora Peterson, $\mathrm{PhD}^{1}$; Deborah M. Stone, ScD ${ }^{2}$; Suzanne M. Marsh, MPA ${ }^{3}$; Pamela K. Schumacher ${ }^{4}$; Hope M. Tiesman, PhD 3 ; \\ Wendy LiKamWa McIntosh, MPH ${ }^{2}$; Colby N. Lokey, MS ${ }^{2}$; Aimée-Rika T. Trudeau, MPH ${ }^{2}$; Brad Bartholow, PhD ${ }^{2}$; Feijun Luo, PhD ${ }^{1}$
}

During 2000-2016, the suicide rate among the U.S. working age population (persons aged 16-64 years) increased $34 \%$, from 12.9 per 100,000 population to 17.3 (https:// www.cdc.gov/injury/wisqars). To better understand suicide among different occupational groups and inform suicide prevention efforts, CDC analyzed suicide deaths by Standard Occupational Classification (SOC) major groups for decedents aged 16-64 years from the 17 states participating in both the 2012 and 2015 National Violent Death Reporting System (NVDRS) (https://www.cdc.gov/violenceprevention/nvdrs). The occupational group with the highest male suicide rate in 2012 and 2015 was Construction and Extraction (43.6 and 53.2 per 100,000 civilian noninstitutionalized working persons, respectively), whereas the group with the highest female suicide rate was Arts, Design, Entertainment, Sports, and Media (11.7 [2012] and 15.6 [2015]). The largest suicide rate increase among males from 2012 to 2015 (47\%) occurred in the Arts, Design, Entertainment, Sports, and Media occupational group (26.9 to 39.7) and among females, in the Food Preparation and Serving Related group, from 6.1 to 9.4 (54\%). CDC's technical package of strategies to prevent suicide is a resource for communities, including workplace settings (1).

NVDRS combines data on all violent deaths (defined as those resulting from the intentional use of physical force or power, threatened or actual, against oneself, another person, or a group or community), including suicide, based on death certificates, coroner/medical examiner reports, and law enforcement reports. Data on usual lifetime occupation among 22,053 suicide decedents aged 16-64 years from the 17 states* that participated in NVDRS in 2012 and 2015 were analyzed. CDC's National Institute for Occupational Safety and Health Industry and Occupation Computerized Coding System (NIOCCS 3.0)

\footnotetext{
*Alaska, Colorado, Georgia, Kentucky, Maryland, Massachusetts, New Jersey, New Mexico, North Carolina, Ohio, Oklahoma, Oregon, Rhode Island, South Carolina, Utah, Virginia, and Wisconsin.
}

(https://wwwn.cdc.gov/nioccs3) was used to assign 2010 U.S. Census civilian occupation and industry codes to NVDRS decedent records based on decedents' usual lifetime occupation and industry as reported on the death certificate. Results are reported by 2010 SOC major groups, converted from U.S. Census codes by NIOCCS. Records that could not be coded by NIOCCS were manually coded using the NIOCCS computer-assisted feature. All coding assignments were reviewed by industry and occupation coding experts for accuracy.

Suicide counts are presented by year, sex, and usual lifetime occupational group. Suicide rates were calculated using annual

\section{INSIDE}

1261 Global Routine Vaccination Coverage — 2017

1265 Progress Toward Global Eradication of Dracunculiasis - January 2017-June 2018

1271 Updated Framework for Development of Evidence-Based Recommendations by the Advisory Committee on Immunization Practices

1273 Increase in Acute Flaccid Myelitis United States, 2018

1276 Notes from the Field: Use of Electronic Cigarettes and Any Tobacco Product Among Middle and High School Students — United States, 2011-2018

1278 Retraction Notice: Suicide Rates by Occupational Group - 17 States, 2012

1279 Correction and Republication: Prevalence and Characteristics of Autism Spectrum Disorder Among Children Aged 8 Years - Autism and Developmental Disabilities Monitoring Network, 11 Sites, United States, 2012

1281 QuickStats

Continuing Education examination available at https://www.cdc.gov/mmwr/cme/conted_info.html\#weekly.

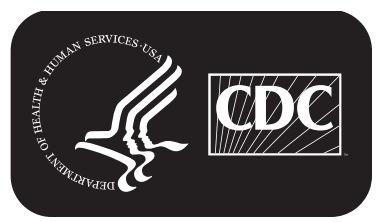

U.S. Department of Health and Human Services Centers for Disease Control and Prevention 
civilian noninstitutionalized working population counts by occupational group (based on longest job held during the previous calendar year) from the Current Population Survey Annual Social and Economic Supplement (2) as the denominator; 95\% confidence intervals (CIs) were calculated using the National Center for Health Statistics method for death rates (3). The rate change from 2012 to 2015 is presented for each occupational group by sex, as is each group's rank for rate change (i.e., where rank position 1 signifies the greatest suicide rate increase). Decedents whose NVDRS data from coroner/ medical examiner reports or law enforcement reports indicated that the decedent was not employed at the time of death (unemployed, disabled, incarcerated, homemaker, or student) were excluded from rate calculations, as were decedents with military or unpaid occupations, and those with insufficient information to classify occupation. Separate analyses of suicide deaths among males in agriculture-related SOC detailed groups were conducted; such rates were not calculated for female decedents because of small numbers.

NIOCCS classified 83\% (8,858 in 2012 and 9,508 in 2015) of decedent records (Table 1); this count includes those that the NIOCCS program determined to have insufficient information to classify occupation. After expert review of NIOCCS automated code assignments, 231 (3\%) of 2012 records and 290 (3\%) of 2015 records were recoded. The remaining 1,799 (2012) and 1,888 (2015) (17\% for both years) records were coded using the NIOCCS computer-assisted feature. For 2012 and 2015 combined, 5,089 (23\%) decedents were not included in suicide rate calculations because they were in the military, had unpaid occupations (e.g., did not work, homemaker, or student), or had insufficient information to classify lifetime occupation. Another 2,236 (10\%) were excluded because they were not employed at the time of death.

In both 2012 and 2015, the largest percentage of male suicides (19\%-20\% of decedents) occurred among those in the Construction and Extraction group (SOC 47) (Table 2); the largest percentage of female suicides in both years occurred among decedents with unpaid occupations (29\%). The largest percentage of female suicides among classifiable occupations occurred in the Office and Administrative Support group (SOC 43) in both years (15\%). In both years, the highest suicide rates among males were in the Construction and Extraction group (43.6 in 2012 and 53.2 in 2015 per 100,000 civilian noninstitutionalized working persons) (Table 3). Among females, the highest suicide rates in both years were in the Arts, Design, Entertainment, Sports, and Media group (SOC 27) (11.7 in 2012 and 15.6 in 2015). Among males, the largest suicide rate increase from 2012 to 2015 (47\%) occurred in the Arts, Design, Entertainment, Sports, and Media group (from 26.9 to 39.7), and among females (54\%) in the Food Preparation and Serving Related group (SOC 35) (from 6.1 to 9.4). Rate changes among females in six SOC major groups were not reported because of small numbers ( $\leq 20$ decedents in one or both years).

The 2012 and 2015 male suicide rates among Farmers, Ranchers, and Other Agricultural Managers (SOC 11-9013,

The MMWR series of publications is published by the Center for Surveillance, Epidemiology, and Laboratory Services, Centers for Disease Control and Prevention (CDC), U.S. Department of Health and Human Services, Atlanta, GA 30329-4027.

Suggested citation: [Author names; first three, then et al., if more than six.] [Report title]. MMWR Morb Mortal Wkly Rep 2018;67:[inclusive page numbers].

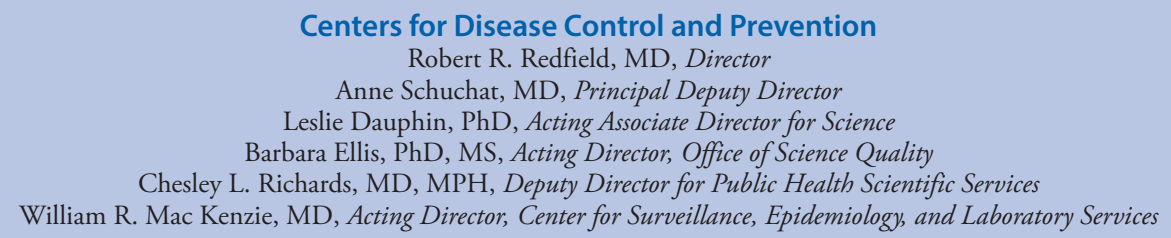

MMWR Editorial and Production Staff (Weekly)

Charlotte K. Kent, PhD, MPH, Acting Editor in Chief, Executive Editor Jacqueline Gindler, MD, Editor

Mary Dott, MD, MPH, Online Editor

Teresa F. Rutledge, Managing Editor

Douglas W. Weatherwax, Lead Technical Writer-Editor Glenn Damon, Soumya Dunworth, PhD, Teresa M. Hood, MS, Technical Writer-Editors

Matthew L. Boulton, MD, MPH Virginia A. Caine, MD

Katherine Lyon Daniel, $\mathrm{PhD}$

Jonathan E. Fielding, MD, MPH, MBA

David W. Fleming, MD

William E. Halperin, MD, DrPH, MPH
MMWR Editorial Board Timothy F. Jones, MD, Chairman Robin Ikeda, MD, MPH Phyllis Meadows, PhD, MSN, RN Jewel Mullen, MD, MPH, MPA Jeff Niederdeppe, PhD Patricia Quinlisk, MD, MPH
Martha F. Boyd, Lead Visual Information Specialist Maureen A. Leahy, Julia C. Martinroe, Stephen R. Spriggs, Tong Yang, Visual Information Specialists Quang M. Doan, MBA, Phyllis H. King, Terraye M. Starr, Moua Yang, Information Technology Specialists
Stephen C. Redd, MD, Patrick L. Remington, MD, MPH Carlos Roig, MS, MA William Schaffner, MD Morgan Bobb Swanson, BS 
Morbidity and Mortality Weekly Report

TABLE 1. Procedure for Identification of analysis cohort of suicide decedents, by occupation - National Violent Death Reporting System (NVDRS) - 17 U.S. states* 2012 and 2015

\begin{tabular}{|c|c|c|}
\hline Analytic procedure & 2012 no. & 2015 no. \\
\hline Suicide decedents obtained from NVDRS data set $^{\dagger}$ & 12,811 & 13,967 \\
\hline \multicolumn{2}{|l|}{ Excluded before assignment of occupation code } & 2,571 \\
\hline \multicolumn{3}{|l|}{ Assignment of occupation code } \\
\hline $\begin{array}{l}\text { Assigned based on decedent usual lifetime } \\
\text { occupation }\end{array}$ & 10,657 & 11,396 \\
\hline Autocoded by NIOCCS & 8,858 & 9,508 \\
\hline $\begin{array}{l}\text { Manually reassigned using the NIOCCS computer- } \\
\text { assisted feature }\end{array}$ & 231 & 290 \\
\hline $\begin{array}{l}\text { Manually assigned using the NIOCCS computer- } \\
\text { assisted feature }\end{array}$ & 1,799 & 1,888 \\
\hline \multicolumn{3}{|l|}{ Rate analysis } \\
\hline $\begin{array}{l}\text { Decedents presumed to be in the labor force at } \\
\text { time of death } \S\end{array}$ & 6,881 & 7,847 \\
\hline
\end{tabular}

Abbreviation: $\mathrm{NIOCCS}=$ National Institute for Occupational Safety and Health Industry and Occupation Computerized Coding System.

* Alaska, Colorado, Georgia, Kentucky, Maryland, Massachusetts, New Jersey, New Mexico, North Carolina, Ohio, Oklahoma, Oregon, Rhode Island, South Carolina, Utah, Virginia, and Wisconsin.

† The total number of deaths (including suicides) reported in the NVDRS data set (June 2018) for the 17 U.S. states analyzed included 19,885 decedents in 2012 and 21,884 decedents in 2015.

$\S$ Because annual U.S. population count data by occupational group reflects the longest held job in the previous year among civilian, noninstitutionalized persons, suicide decedents whose NVDRS data from coroner/medical examiner or law enforcement reports indicated no employment (e.g., retired, unemployed, disabled, incarcerated, home maker, or student) at the time of death were excluded from rate analysis. Decedents were excluded if NVDRS current occupation information contained any of the following: "student, unemp*, not empl*, laid off, retir*, disab*, incarcer*, inmate, prisoner, homemaker, home maker, housewife, house wife, never worked, or not working." Manual review of records excluded based on these criteria resulted in five records being reinstated (e.g., "student teacher" and two occupations listed for a decedent with just one occupation explicitly identified as retired).

a subgroup of the SOC 11 Management major group) were $44.9(\mathrm{CI}=34.2-57.9)$ and $32.2(\mathrm{CI}=24.2-42.0)$ per 100,000 , based on 59 and 54 suicides in 2012 and 2015, respectively. The 2012 and 2015 male suicide rates for Agricultural Workers (SOC 45-2000, a subgroup of the SOC 45 Farming, Fishing, and Forestry major group) were $20.4(\mathrm{CI}=13.8-29.1)$ and $17.3(\mathrm{CI}=12.1-23.9)$, based on 30 and 36 suicides in 2012 and 2015, respectively.

\section{Discussion}

Suicide rates varied widely across occupational groups in both 2012 and 2015, and rates among males and females increased in many occupational groups. The etiology of suicide is multifactorial, and identifying the specific role that occupational factors might play in suicide risk is complicated; both work (e.g., little job control or job insecurity) and nonwork (e.g., relationship conflict) factors are associated with psychological distress and suicide (4). The relationship between occupation and suicide might be confounded by access to lethal means on the job and socioeconomic factors such as lower income
TABLE 2. Number and percentage of suicide decedents* in Standard Occupational Classification (SOC) major group, by year and sex National Violent Death Reporting System, 17 states, ${ }^{\dagger} 2012$ and 2015

\begin{tabular}{|c|c|c|c|c|c|}
\hline \multirow{2}{*}{$\begin{array}{l}\text { SOC } \\
\text { code }\end{array}$} & \multirow[b]{2}{*}{ Occupational group } & \multicolumn{2}{|c|}{ Male } & \multicolumn{2}{|c|}{ Female } \\
\hline & & $\begin{array}{l}2012 \\
\text { no. }(\%)\end{array}$ & $\begin{array}{l}2015 \\
\text { no. }(\%)\end{array}$ & $\begin{array}{l}2012 \\
\text { no. }(\%)\end{array}$ & $\begin{array}{l}2015 \\
\text { no. }(\%)\end{array}$ \\
\hline 11 & Management & $534(8)$ & $611(9)$ & $117(7)$ & $118(7)$ \\
\hline 13 & $\begin{array}{l}\text { Business and Financial } \\
\text { Operations }\end{array}$ & $155(2)$ & $145(2)$ & $81(5)$ & $84(5)$ \\
\hline 15 & Computer and Mathematical & $208(3)$ & $237(3)$ & $22(1)$ & $32(2)$ \\
\hline 17 & Architecture and Engineering & $172(3)$ & $167(2)$ & $10(1)$ & $15(1)$ \\
\hline 19 & Life, Physical, and Social Science & $56(1)$ & $52(1)$ & $15(1)$ & $21(1)$ \\
\hline 21 & Community and Social Service & $41(1)$ & $48(1)$ & $39(2)$ & $40(2)$ \\
\hline 23 & Legal & $54(1)$ & $49(1)$ & $34(2)$ & $29(2)$ \\
\hline 25 & Education, Training, and Library & $91(1)$ & $87(1)$ & $82(5)$ & $84(5)$ \\
\hline 27 & $\begin{array}{l}\text { Arts, Design, Entertainment, } \\
\text { Sports, and Media }\end{array}$ & $140(2)$ & $186(3)$ & $54(3)$ & $76(4)$ \\
\hline 29 & $\begin{array}{l}\text { Health Care Practitioners and } \\
\text { Technical occupations }\end{array}$ & $145(2)$ & $169(2)$ & $220(14)$ & $225(12)$ \\
\hline 31 & Health Care Support & $35(1)$ & $34(<1)$ & $97(6)$ & $124(7)$ \\
\hline 33 & Protective Service & $232(4)$ & $226(3)$ & $29(2)$ & $32(2)$ \\
\hline 35 & $\begin{array}{l}\text { Food Preparation and } \\
\text { Serving Related }\end{array}$ & $214(3)$ & $301(4)$ & $112(7)$ & $154(9)$ \\
\hline 37 & $\begin{array}{l}\text { Building and Grounds } \\
\text { Cleaning and Maintenance }\end{array}$ & $316(5)$ & $315(4)$ & $36(2)$ & $46(3)$ \\
\hline 39 & Personal Care and Service & $81(1)$ & $85(1)$ & $98(6)$ & $102(6)$ \\
\hline 41 & Sales and Related & $555(9)$ & $553(8)$ & $170(11)$ & $212(12)$ \\
\hline 43 & $\begin{array}{l}\text { Office and Administrative } \\
\text { Support }\end{array}$ & $244(4)$ & $260(4)$ & $234(15)$ & $268(15)$ \\
\hline 45 & Farming, Fishing, and Forestry & $68(1)$ & $71(1)$ & $7(<1)$ & $5(<1)$ \\
\hline 47 & Construction and Extraction & $1,216(19)$ & $1,404(20)$ & $12(1)$ & $17(1)$ \\
\hline 49 & $\begin{array}{l}\text { Installation, Maintenance, and } \\
\text { Repair }\end{array}$ & 549 (9) & $621(9)$ & $8(1)$ & NR \\
\hline 51 & Production & $605(9)$ & $679(10)$ & $64(4)$ & $81(4)$ \\
\hline 53 & $\begin{array}{l}\text { Transportation and } \\
\text { Material Moving }\end{array}$ & $736(11)$ & $817(11)$ & $52(3)$ & $39(2)$ \\
\hline NA & Military & $228(3)$ & $203(2)$ & $15(1)$ & $13(<1)$ \\
\hline NA & Unpaid & $822(10)$ & $913(11)$ & 724 (29) & 795 (29) \\
\hline NA & $\begin{array}{l}\text { Insufficient Information to } \\
\text { Classify Occupation }\end{array}$ & $651(8)$ & $425(5)$ & 177 (9) & $123(4)$ \\
\hline
\end{tabular}

Abbreviation: $\mathrm{NA}=$ not assigned; $\mathrm{NR}=$ not reported due to cell size $<5$.

* Aged 16-64 years.

† Alaska, Colorado, Georgia, Kentucky, Maryland, Massachusetts, New Jersey, New Mexico, North Carolina, Ohio, Oklahoma, Oregon, Rhode Island, South Carolina, Utah, Virginia, and Wisconsin.

and education $(5,6)$. Previous studies have employed a range of methodologies to study the proposed association between suicide and occupation and, at times, have arrived at different conclusions. For example, although this analysis aligns with another that found high suicide rates among construction workers in Colorado (7), a meta-analysis using an international occupational classification system found persons in other less-skilled occupations, such as laborers and cleaners, to be at higher risk (G).

A better understanding of how suicides are distributed by occupational group might help inform prevention programs and policies. Because many adults spend a substantial amount of their time at work, the workplace is an important but underutilized location for suicide prevention (8). Workplaces could potentially benefit from suicide prevention activities. 
TABLE 3. Suicide rate per 100,000 civilian, noninstitutionalized working persons aged 16-64 years, by sex, based on suicide decedents $(\mathrm{N}=14,728)$ presumed in the labor force at time of death using Standard Occupational Classification (SOC) major groups - National Violent Death Reporting System, 17 states, ${ }^{*} 2012$ and 2015

\begin{tabular}{|c|c|c|c|c|c|c|c|c|c|c|c|}
\hline \multicolumn{6}{|c|}{ Males } & \multicolumn{6}{|c|}{ Females } \\
\hline \multirow{2}{*}{$\begin{array}{l}\text { SOC } \\
\text { code }\end{array}$} & \multirow[b]{2}{*}{ Occupational group } & \multirow[b]{2}{*}{2012} & \multirow[b]{2}{*}{2015} & \multicolumn{2}{|c|}{ Rate change } & \multirow{2}{*}{$\begin{array}{l}\text { SOC } \\
\text { code }\end{array}$} & \multirow[b]{2}{*}{ Occupational group } & \multirow[b]{2}{*}{2012} & \multirow[b]{2}{*}{2015} & \multicolumn{2}{|c|}{ Rate change } \\
\hline & & & & $\%$ & Rank $^{\dagger}$ & & & & & $\%$ & Rank $^{\dagger}$ \\
\hline \multirow[t]{6}{*}{47} & \multicolumn{5}{|l|}{ Construction and Extraction } & \multirow[t]{6}{*}{27} & \multicolumn{5}{|c|}{ Arts, Design, Entertainment, Sports, and Media } \\
\hline & Rate rank ${ }^{\S}$ & 1 & 1 & $+22 \%$ & 5 & & Rate rank ${ }^{\S}$ & 1 & 1 & $+34 \%$ & 2 \\
\hline & Rate per 100,000 & 43.6 & 53.2 & & & & Rate per 100,000 & 11.7 & 15.6 & & \\
\hline & $95 \% \mathrm{Cl}^{9}$ & $40.9-46.3$ & $50.2-56.1$ & & & & $95 \% \mathrm{Cl}^{\mathbb{9}}$ & $8.6-15.5$ & $12.1-19.8$ & & \\
\hline & Suicide decedents, no. & 1,009 & 1,248 & & & & Suicide decedents, no. & 47 & 67 & & \\
\hline & Population, no. & $2,313,934$ & $2,345,952$ & & & & Population, no. & 403,305 & 429,424 & & \\
\hline \multirow[t]{6}{*}{27} & \multicolumn{5}{|c|}{ Arts, Design, Entertainment, Sports, and Media } & 33 & Protective Service & & & & \\
\hline & Rate rank & 7 & 2 & $+47 \%$ & 1 & & Rate rank & 2 & 2 & $+5 \%$ & 9 \\
\hline & Rate per 100,000 & 26.9 & 39.7 & & & & Rate per 100,000 & 11.6 & 12.2 & & \\
\hline & $95 \% \mathrm{Cl}$ & $22.1-31.8$ & $33.6-45.8$ & & & & $95 \% \mathrm{Cl}$ & $7.5-17.1$ & $8.1-17.7$ & & \\
\hline & Suicide decedents, no. & 117 & 162 & & & & Suicide decedents, no. & 25 & 28 & & \\
\hline & Population, no. & 434,177 & 408,113 & & & & Population, no. & 215,345 & 228,862 & & \\
\hline 49 & Installation, Maintenance & d Repair & & & & 31 & Health Care Support & & & & \\
\hline & Rate rank & 2 & 3 & $+24 \%$ & 3 & & Rate rank & 5 & 3 & $+31 \%$ & 3 \\
\hline & Rate per 100,000 & 31.6 & 39.1 & & & & Rate per 100,000 & 8.4 & 11.0 & & \\
\hline & $95 \% \mathrm{Cl}$ & $28.7-34.4$ & $35.8-42.3$ & & & & $95 \% \mathrm{Cl}$ & $6.7-10.4$ & $8.9-13.0$ & & \\
\hline & Suicide decedents, no. & 473 & 542 & & & & Suicide decedents, no. & 83 & 108 & & \\
\hline & Population, no. & $1,498,263$ & $1,387,681$ & & & & Population, no. & 993,407 & 984,369 & & \\
\hline 53 & Transportation and Mater & Moving & & & & 35 & Food Preparation and Serv & Related & & & \\
\hline & Rate rank & 4 & 4 & $+9 \%$ & 8 & & Rate rank & 11 & 4 & $+54 \%$ & 1 \\
\hline & Rate per 100,000 & 28.4 & 30.9 & & & & Rate per 100,000 & 6.1 & 9.4 & & \\
\hline & $95 \% \mathrm{Cl}$ & $26.2-30.7$ & $28.6-33.1$ & & & & $95 \% \mathrm{Cl}$ & $4.9-7.5$ & $7.8-11.0$ & & \\
\hline & Suicide decedents, no. & 615 & 721 & & & & Suicide decedents, no. & 94 & 139 & & \\
\hline & Population, no. & $2,164,530$ & $2,336,133$ & & & & Population, no. & $1,539,199$ & $1,479,822$ & & \\
\hline 51 & Production & & & & & 23 & Legal & & & & \\
\hline & Rate rank & 3 & 5 & $+7 \%$ & 10 & & Rate rank & 3 & 5 & $-17 \%$ & 15 \\
\hline & Rate per 100,000 & 28.4 & 30.5 & & & & Rate per 100,000 & 11.1 & 9.2 & & \\
\hline & $95 \% \mathrm{Cl}$ & $26.0-30.9$ & $28.1-33.0$ & & & & $95 \% \mathrm{Cl}$ & $7.5-15.9$ & $5.8-13.9$ & & \\
\hline & Suicide decedents, no. & 524 & 607 & & & & Suicide decedents, no. & 30 & 22 & & \\
\hline & Population, no. & $1,843,879$ & $1,987,864$ & & & & Population, no. & 269,243 & 238,870 & & \\
\hline 33 & Protective Service & & & & & 29 & Health Care Practitioners a & echnical & & & \\
\hline & Rate rank & 6 & 6 & $+4 \%$ & 11 & & Rate rank & 4 & 6 & $-13 \%$ & 13 \\
\hline & Rate per 100,000 & 27.1 & 28.2 & & & & Rate per 100,000 & 10.3 & 9.0 & & \\
\hline & $95 \% \mathrm{Cl}$ & $23.3-30.9$ & $24.2-32.1$ & & & & $95 \% \mathrm{Cl}$ & $8.9-11.8$ & $7.7-10.3$ & & \\
\hline & Suicide decedents, no. & 198 & 194 & & & & Suicide decedents, no. & 195 & 193 & & \\
\hline & Population, no. & 730,044 & 689,034 & & & & Population, no. & $1,890,885$ & $2,140,217$ & & \\
\hline 37 & Building and Grounds Cle & g and Maint & ance & & & 51 & Production & & & & \\
\hline & Rate rank & 5 & 7 & $-2 \%$ & 14 & & Rate rank & 7 & 7 & $+18 \%$ & 6 \\
\hline & Rate per 100,000 & 27.3 & 26.8 & & & & Rate per 100,000 & 7.6 & 9.0 & & \\
\hline & $95 \% \mathrm{Cl}$ & $24.1-30.5$ & $23.6-30.0$ & & & & $95 \% \mathrm{Cl}$ & $5.8-10.0$ & $7.0-11.3$ & & \\
\hline & Suicide decedents, no. & 281 & 276 & & & & Suicide decedents, no. & 55 & 72 & & \\
\hline & Population, no. & $1,028,779$ & $1,029,385$ & & & & Population, no. & 719,183 & 800,640 & & \\
\hline 29 & Health Care Practitioners & Technical & & & & 39 & Personal Care and Service & & & & \\
\hline & Rate rank & 14 & 8 & $+23 \%$ & 4 & & Rate rank & 9 & 8 & $+14 \%$ & 7 \\
\hline & Rate per 100,000 & 20.8 & 25.6 & & & & Rate per 100,000 & 6.8 & 7.7 & & \\
\hline & $95 \% \mathrm{Cl}$ & $17.1-24.6$ & $21.5-29.8$ & & & & $95 \% \mathrm{Cl}$ & $5.5-8.4$ & $6.2-9.5$ & & \\
\hline & Suicide decedents, no. & 119 & 145 & & & & Suicide decedents, no. & 89 & 92 & & \\
\hline & Population, no. & 571,387 & 565,768 & & & & Population, no. & $1,308,535$ & $1,187,811$ & & \\
\hline
\end{tabular}

See table footnotes on page 1258. 
TABLE 3. (Continued) Suicide rate per 100,000 civilian, noninstitutionalized working persons aged $16-64$ years, by sex, based on suicide decedents $(\mathrm{N}=14,728)$ presumed in the labor force at time of death using Standard Occupational Classification (SOC) major groups - National Violent Death Reporting System, 17 states, $^{*} 2012$ and 2015

\begin{tabular}{|c|c|c|c|c|c|c|c|c|c|c|c|}
\hline & & Males & & & & & Fen & nales & & & \\
\hline & & & & Rate c & hange & & & & & Rate c & hange \\
\hline code & Occupational group & 2012 & 2015 & $\%$ & $\operatorname{Rank}^{\dagger}$ & code & Occupational group & 2012 & 2015 & $\%$ & $\operatorname{Rank}^{\dagger}$ \\
\hline 45 & Farming, Fishing, and Forestry & & & & & 41 & Sales and Related & & & & \\
\hline & Rate rank & 8 & 9 & $-13 \%$ & 21 & & Rate rank & 10 & 9 & $+20 \%$ & 5 \\
\hline & Rate per 100,000 & 26.3 & 22.8 & & & & Rate per 100,000 & 6.4 & 7.7 & & \\
\hline & $95 \% \mathrm{Cl}$ & $20.0-34.0$ & $17.7-29.0$ & & & & $95 \% \mathrm{Cl}$ & $5.3-7.4$ & $6.6-8.7$ & & \\
\hline & Suicide decedents, no. & 58 & 67 & & & & Suicide decedents, no. & 148 & 192 & & \\
\hline & Population, no. & 220,364 & 293,746 & & & & Population, no. & $2,325,223$ & $2,505,186$ & & \\
\hline 41 & $\begin{array}{l}\text { Sales and Related } \\
\text { Rate rank }\end{array}$ & 11 & 10 & $+1 \%$ & 12 & 15 & $\begin{array}{l}\text { Computer and Mathematical } \\
\text { Rate rank }\end{array}$ & NR & 10 & NR & NR \\
\hline & Rate per 100,000 & 21.3 & 21.5 & & & & Rate per 100,000 & NR & 7.3 & & \\
\hline & $95 \% \mathrm{Cl}$ & $19.4-23.2$ & $19.6-23.4$ & & & & $95 \% \mathrm{Cl}$ & NR & $5.0-10.5$ & & \\
\hline & Suicide decedents, no. & 487 & 489 & & & & Suicide decedents, no. & 20 & 30 & & \\
\hline & Population, no. & $2,282,361$ & $2,276,666$ & & & & Population, no. & 390,260 & 408,410 & & \\
\hline 35 & Food Preparation and Serving & Related & & & & 53 & Transportation and Material Mo & ving & & & \\
\hline & Rate rank & 19 & 11 & $+43 \%$ & 2 & & Rate rank & 6 & 11 & $-17 \%$ & 14 \\
\hline & Rate per 100,000 & 14.6 & 20.9 & & & & Rate per 100,000 & 8.3 & 6.9 & & \\
\hline & $95 \% \mathrm{Cl}$ & $12.5-16.7$ & $18.4-23.3$ & & & & $95 \% \mathrm{Cl}$ & $6.0-11.2$ & $4.8-9.7$ & & \\
\hline & Suicide decedents, no. & 180 & 276 & & & & Suicide decedents, no. & 43 & 33 & & \\
\hline & Population, no. & $1,234,381$ & $1,321,800$ & & & & Population, no. & 517,082 & 477,143 & & \\
\hline 31 & Health Care Support & & & & & 21 & Community and Social Service & & & & \\
\hline & Rate rank & 9 & 12 & $-12 \%$ & 18 & & Rate rank & 8 & 12 & $-17 \%$ & 16 \\
\hline & Rate per 100,000 & 22.1 & 19.5 & & & & Rate per 100,000 & 7.3 & 6.0 & & \\
\hline & $95 \% \mathrm{Cl}$ & $14.8-31.7$ & $12.5-29.0$ & & & & $95 \% \mathrm{Cl}$ & $5.1-10.2$ & $4.2-8.4$ & & \\
\hline & Suicide decedents, no. & 29 & 24 & & & & Suicide decedents, no. & 34 & 36 & & \\
\hline & Population, no. & 131,497 & 123,003 & & & & Population, no. & 464,942 & 595,582 & & \\
\hline 17 & Architecture and Engineering & & & & & 43 & Office and Administrative Supp & & & & \\
\hline & Rate rank & 10 & 13 & $-10 \%$ & 15 & & Rate rank & 14 & 13 & $+27 \%$ & 4 \\
\hline & Rate per 100,000 & 21.6 & 19.4 & & & & Rate per 100,000 & 4.7 & 6.0 & & \\
\hline & $95 \% \mathrm{Cl}$ & $18.1-25.1$ & $16.3-22.6$ & & & & $95 \% \mathrm{Cl}$ & $4.1-5.4$ & $5.2-6.8$ & & \\
\hline & Suicide decedents, no. & 145 & 147 & & & & Suicide decedents, no. & 201 & 239 & & \\
\hline & Population, no. & 670,938 & 756,515 & & & & Population, no. & $4,267,892$ & $3,985,105$ & & \\
\hline 23 & Legal & & & & & 13 & Business and Financial Operatio & & & & \\
\hline & Rate rank & 12 & 14 & $-12 \%$ & 19 & & Rate rank & 12 & 14 & $-5 \%$ & 11 \\
\hline & Rate per 100,000 & 21.3 & 18.7 & & & & Rate per 100,000 & 5.7 & 5.4 & & \\
\hline & $95 \% \mathrm{Cl}$ & $15.7-28.2$ & $13.4-25.4$ & & & & $95 \% \mathrm{Cl}$ & $4.4-7.2$ & $4.2-6.8$ & & \\
\hline & Suicide decedents, no. & 48 & 41 & & & & Suicide decedents, no. & 70 & 71 & & \\
\hline & Population, no. & 225,681 & 219,171 & & & & Population, no. & $1,235,880$ & $1,321,724$ & & \\
\hline 11 & Management & & & & & 37 & Building and Grounds Cleaning & and Mainte & nance & & \\
\hline & Rate rank & 17 & 15 & $+8 \%$ & 9 & & Rate rank & 15 & 15 & $+14 \%$ & 8 \\
\hline & Rate per 100,000 & 16.4 & 17.8 & & & & Rate per 100,000 & 4.6 & 5.2 & & \\
\hline & $95 \% \mathrm{Cl}$ & $14.9-17.9$ & $16.3-19.3$ & & & & $95 \% \mathrm{Cl}$ & $3.1-6.5$ & $3.7-7.2$ & & \\
\hline & Suicide decedents, no. & 477 & 530 & & & & Suicide decedents, no. & 31 & 36 & & \\
\hline & Population, no. & $2,906,468$ & $2,981,498$ & & & & Population, no. & 673,483 & 688,809 & & \\
\hline 39 & Personal Care and Service & & & & & 11 & Management & & & & \\
\hline & Rate rank & 13 & 16 & $-21 \%$ & 22 & & Rate rank & 13 & 16 & $-12 \%$ & 12 \\
\hline & Rate per 100,000 & 20.9 & 16.5 & & & & Rate per 100,000 & 5.6 & 4.9 & & \\
\hline & $95 \% \mathrm{Cl}$ & $16.2-26.4$ & $12.9-20.7$ & & & & $95 \% \mathrm{Cl}$ & $4.5-6.7$ & $4.0-5.9$ & & \\
\hline & Suicide decedents, no. & 68 & 73 & & & & Suicide decedents, no. & 104 & 103 & & \\
\hline & Population, no. & 326,037 & 443,543 & & & & Population, no. & $1,855,055$ & $2,083,968$ & & \\
\hline
\end{tabular}

See table footnotes on page 1258. 
TABLE 3. (Continued) Suicide rate per 100,000 civilian, noninstitutionalized working persons aged $16-64$ years, by sex, based on suicide decedents $(\mathrm{N}=14,728)$ presumed in the labor force at time of death using Standard Occupational Classification (SOC) major groups — National Violent Death Reporting System, 17 states, $^{*} 2012$ and 2015

\begin{tabular}{|c|c|c|c|c|c|c|c|c|c|c|c|}
\hline \multicolumn{6}{|c|}{ Males } & \multicolumn{6}{|c|}{ Females } \\
\hline \multirow{2}{*}{$\begin{array}{l}\text { SOC } \\
\text { code }\end{array}$} & \multirow[b]{2}{*}{ Occupational group } & \multirow[b]{2}{*}{2012} & \multirow[b]{2}{*}{2015} & \multicolumn{2}{|c|}{ Rate change } & \multirow{2}{*}{$\begin{array}{l}\text { SOC } \\
\text { code }\end{array}$} & \multirow[b]{2}{*}{ Occupational group } & \multirow[b]{2}{*}{2012} & \multirow[b]{2}{*}{2015} & \multicolumn{2}{|c|}{ Rate change } \\
\hline & & & & $\%$ & $\operatorname{Rank}^{\dagger}$ & & & & & $\%$ & Rank $^{\dagger}$ \\
\hline \multirow[t]{6}{*}{15} & \multicolumn{5}{|c|}{ Computer and Mathematical } & 25 & \multicolumn{5}{|l|}{ Education, Training, and Library } \\
\hline & Rate rank & 15 & 17 & $-11 \%$ & 16 & & Rate rank & 16 & 17 & $+3 \%$ & 10 \\
\hline & Rate per 100,000 & 18.1 & 16.1 & & & & Rate per 100,000 & 3.3 & 3.4 & & \\
\hline & $95 \% \mathrm{Cl}$ & $15.5-20.8$ & $13.9-18.4$ & & & & $95 \% \mathrm{Cl}$ & $2.6-4.2$ & $2.7-4.2$ & & \\
\hline & Suicide decedents, no. & 179 & 202 & & & & Suicide decedents, no. & 69 & 74 & & \\
\hline & Population, no. & 986,994 & $1,252,275$ & & & & Population, no. & $2,091,706$ & $2,186,483$ & & \\
\hline \multirow[t]{6}{*}{43} & \multicolumn{5}{|c|}{ Office and Administrative Support } & 17 & \multicolumn{5}{|l|}{ Architecture and Engineering } \\
\hline & Rate rank & 20 & 18 & $+12 \%$ & 7 & & Rate rank & NR & NR & NR & NR \\
\hline & Rate per 100,000 & 14.1 & 15.8 & & & & Rate per 100,000 & NR & NR & & \\
\hline & $95 \% \mathrm{Cl}$ & $12.2-16.1$ & $13.7-17.9$ & & & & $95 \% \mathrm{Cl}$ & NR & NR & & \\
\hline & Suicide decedents, no. & 206 & 223 & & & & Suicide decedents, no. & 10 & 12 & & \\
\hline & Population, no. & $1,456,242$ & $1,411,453$ & & & & Population, no. & 135,632 & 144,852 & & \\
\hline \multirow[t]{6}{*}{19} & \multicolumn{5}{|c|}{ Life, Physical, and Social Science } & 19 & \multicolumn{5}{|l|}{ Life, Physical, and Social Science } \\
\hline & Rate rank & 16 & 19 & $-13 \%$ & 20 & & Rate rank & NR & NR & NR & NR \\
\hline & Rate per 100,000 & 17.3 & 15.0 & & & & Rate per 100,000 & NR & NR & & \\
\hline & $95 \% \mathrm{Cl}$ & $12.7-23.0$ & $11.0-20.0$ & & & & $95 \% \mathrm{Cl}$ & NR & NR & & \\
\hline & Suicide decedents, no. & 47 & 47 & & & & Suicide decedents, no. & 13 & 19 & & \\
\hline & Population, no. & 271,690 & 312,925 & & & & Population, no. & 225,992 & 204,566 & & \\
\hline \multirow[t]{6}{*}{21} & \multicolumn{5}{|c|}{ Community and Social Service } & 45 & \multicolumn{5}{|l|}{ Farming, Fishing, and Forestry } \\
\hline & Rate rank & 21 & 20 & $+15 \%$ & 6 & & Rate rank & NR & NR & NR & NR \\
\hline & Rate per 100,000 & 12.8 & 14.6 & & & & Rate per 100,000 & NR & NR & & \\
\hline & $95 \% \mathrm{Cl}$ & $8.8-17.9$ & $10.7-19.6$ & & & & $95 \% \mathrm{Cl}$ & NR & NR & & \\
\hline & Suicide decedents, no. & 33 & 45 & & & & Suicide decedents, no. & 7 & 5 & & \\
\hline & Population, no. & 258,744 & 307,829 & & & & Population, no. & 54,068 & 91,967 & & \\
\hline 13 & Business and Financial $\mathrm{O}$ & tions & & & & 47 & Construction and Extraction & & & & \\
\hline & Rate rank & 18 & 21 & $-11 \%$ & 17 & & Rate rank & NR & NR & NR & NR \\
\hline & Rate per 100,000 & 14.6 & 13.0 & & & & Rate per 100,000 & NR & NR & & \\
\hline & $95 \% \mathrm{Cl}$ & $12.1-17.2$ & $10.7-15.3$ & & & & $95 \% \mathrm{Cl}$ & $\mathrm{NR}$ & NR & & \\
\hline & Suicide decedents, no. & 125 & 122 & & & & Suicide decedents, no. & 9 & 14 & & \\
\hline & Population, no. & 855,329 & 941,806 & & & & Population, no. & 55,164 & 76,173 & & \\
\hline 25 & Education, Training, and & & & & & 49 & Installation, Maintenance, and & Repair & & & \\
\hline & Rate rank & 22 & 22 & $-1 \%$ & 13 & & Rate rank & NR & NR & NR & NR \\
\hline & Rate per 100,000 & 10.9 & 10.9 & & & & Rate per 100,000 & NR & NR & & \\
\hline & $95 \% \mathrm{Cl}$ & $8.6-13.6$ & $8.6-13.5$ & & & & $95 \% \mathrm{Cl}$ & NR & NR & & \\
\hline & Suicide decedents, no. & 78 & 79 & & & & Suicide decedents, no. & 8 & NR & & \\
\hline & Population, no. & 713,321 & 727,167 & & & & Population, no. & 73,231 & 46,136 & & \\
\hline
\end{tabular}

Abbreviations: $\mathrm{Cl}=$ confidence interval; $\mathrm{NR}=$ not reported; number of decedents not reported $<5$, and rates were not calculated for occupational groups with $\leq 20$ decedents; SOC $=$ Standard Occupational Classification.

† Rate change rank refers to each occupational group's rank order for rate change from 2012 to 2015, where rank position 1 signifies the greatest suicide rate increase.

$\S$ Occupational groups were ranked by 2015 suicide rate, separately for males and females. Because of rounding, some rate and rate change results are not precisely calculable from the data presented.

9 95\% Cls were calculated using CDC's National Center for Health Statistics methods, including confidence limit factors for mortality rates based on $<100$ decedents.

Additional and tailored prevention approaches might be necessary to support workers at higher risk. Workplace suicide prevention efforts to date have focused primarily on early detection and tertiary intervention through the training of persons (i.e. gatekeepers) to identify those at risk for suicide and refer them to supporting services. However, more research on the role of the workplace in primary suicide prevention is needed, including improving working conditions and reducing stress (8).

The findings in this report are subject to at least four limitations. First, because of the nature of the data that were available, and consistent with previous research methods, this report compared decedents' usual lifetime occupation as recorded on the death certificate with occupations of the employed population to calculate suicide rates. Additional 


\section{Summary}

What is already known about this topic?

From 2000 to 2016, the U.S. suicide rate among working aged (16-64 years) adults increased 34\% from 12.9 per 100,000 population to 17.3 .

What is added by this report?

2012 and 2015 National Violent Death Reporting System data from 17 states indicated the major occupational group with the highest male suicide rate was Construction and Extraction (43.6 [2012] and 53.2 [2015]). The Arts, Design, Entertainment, Sports, and Media major occupation group had the highest female suicide rate in 2012 (11.7) and 2015 (15.6).

What are the implications for public health practice?

A comprehensive approach to suicide prevention, including workplace-based approaches, is needed. CDC's technical package of strategies to prevent suicide is a resource for communities and workplaces to identify prevention strategies with the best available evidence.

data from coroner/medical examiner and law enforcement reports were used to exclude decedents identified as not in the labor force at time of death. Separate analyses indicated that if no such exclusion were applied, suicide rates would have been higher for all groups, although the top and bottom eight ranked occupational groups in 2015 by male suicide rate would maintain the same rank position, as would the top three and bottom four occupational groups by female suicide rate. Second, this report did not address confounding factors that might account for higher or lower rates of suicide between and within occupational groups, including education and income $(9,10)$. Within SOC major occupational groups, employee education and income might vary widely. For example, the Management SOC major group includes farmers, ranchers, and chief executives of large companies, and the Construction and Extraction group includes both employees who might be salaried (e.g., supervisors) and those who might be paid hourly wages (e.g., roofer helpers). Future research might benefit from using more narrowly defined occupational groups and controlling for education and income to refine understanding of the relationship between occupation and suicide. Third, industry and occupation data obtained from death certificates rely on the accuracy and completeness of employment information provided by decedents' family members. It is also possible that completeness and accuracy of that information might be associated with decedents' job history. For example, categorization based on single lifetime industry and occupation might not accurately reflect employment for those persons with multiple lifetime occupations and those who worked across industries. Finally, this report is based on data from 17 U.S. states that participated in NVDRS in 2012 and 2015, and the data are not nationally representative.

To address the multifactorial etiology of suicide, CDC recommends a comprehensive approach to prevention (1). Strategies might include enhancing social connectedness and expanding access to relevant resources, strengthening state or local economic supports, implementing practices that encourage help-seeking and decrease stigma, and providing referrals to mental health and other services (1). Strategies can be implemented to assure support and reduce access to lethal means among persons at risk. Decision makers, including employers, can create a response plan, should a suicide affect their organization. Surviving family and friends can be supported to reduce their own suicide risk. The media can follow reporting recommendations to avoid sensationalized reporting and can refrain from providing details on suicide methods (1). Further workplace prevention resources are available at https://theactionalliance.org/, and help is available at 1-800-273-TALK.

\section{Acknowledgments}

Jeff Purdin, Matt Hirst, Susan Burton, Jenny Huddleston, Elizabeth Smith, Division of Surveillance, Hazard Evaluations, and Field Studies, National Institute for Occupational Safety and Health, CDC.

Corresponding author: Deborah M. Stone, dstone3@cdc.gov, 770-488-3942.

\footnotetext{
${ }^{1}$ Division of Analysis, Research, and Practice Integration, National Center for Injury Prevention and Control, CDC; ${ }^{2}$ Division of Violence Prevention, National Center for Injury Prevention and Control, CDC; ${ }^{3}$ Division of Safety Research, National Institute for Occupational Safety and Health, CDC; ${ }^{4}$ Division of Surveillance, Hazard Evaluations, and Field Studies, National Institute for Occupational Safety and Health, CDC.
}

All authors have completed and submitted the ICMJE form for disclosure of potential conflicts of interest. No potential conflicts of interest were disclosed.

\section{References}

1. Stone DM, Holland K, Bartholow B, Crosby A, Davis S, Wilkins N. Preventing suicide: a technical package of policies, programs, and practices. Atlanta, GA: US Department of Health and Human Services, CDC; 2017. https://www.cdc.gov/violenceprevention/pdf/ suicideTechnicalPackage.pdf

2. US Census Bureau. Current population survey annual social and economic supplement 2013 and 2016. Washington, DC: US Census Bureau; 2018.

3. National Center for Health Statistics. Vital statistics of the United States: mortality, 1999 technical appendix. Hyattsville, MD: US Department of Health and Human Services, CDC, National Center for Health Statistics; 2004. https://www.cdc.gov/nchs/data/statab/techap99.pdf

4. Milner A, Witt K, LaMontagne AD, Niedhammer I. Psychosocial job stressors and suicidality: a meta-analysis and systematic review. Occup Environ Med 2018;75:245-53. https://doi.org/10.1136/ oemed-2017-104531 
5. Milner A, Witt K, Maheen H, LaMontagne AD. Access to means of suicide, occupation and the risk of suicide: a national study over 12 years of coronial data. BMC Psychiatry 2017;17:125. https://doi.org/10.1186/ s12888-017-1288-0

6. Milner A, Spittal MJ, Pirkis J, LaMontagne AD. Suicide by occupation: systematic review and meta-analysis. Br J Psychiatry 2013;203:409-16. https://doi.org/10.1192/bjp.bp.113.128405

7. Stallones L, Doenges T, Dik BJ, Valley MA. Occupation and suicide: Colorado, 2004-2006. Am J Ind Med 2013;56:1290-5.
8. Milner A, Page K, Spencer-Thomas S, Lamotagne AD. Workplace suicide prevention: a systematic review of published and unpublished activities. Health Promot Int 2015;30:29-37. https://doi.org/10.1093/heapro/ dau085

9. Daly MC, Wilson DJ, Johnson NJ. Relative status and well-being: evidence from U.S. suicide deaths. Rev Econ Stat 2013;95:1480-500. https://doi.org/10.1162/REST_a_00355

10. Phillips JA, Hempstead K. Differences in U.S. suicide rates by educational attainment, 2000-2014. Am J Prev Med 2017;53:e123-30. https://doi.org/10.1016/j.amepre.2017.04.010 